\title{
Newly Diagnosed Celiac Disease and Bone Health in Young Adults: A Systematic Literature Review
}

\author{
Caterina Mosca ${ }^{1,2} \cdot$ Fanney Thorsteinsdottir ${ }^{3}$ Bo Abrahamsen ${ }^{1,4,5} \cdot$ Jüri Johannes Rumessen ${ }^{6}$. \\ Mina Nicole Händel ${ }^{3}$ (10
}

Received: 25 October 2021 / Accepted: 18 December 2021 / Published online: 3 January 2022

(c) The Author(s), under exclusive licence to Springer Science+Business Media, LLC, part of Springer Nature 2022

\begin{abstract}
Celiac disease (CD), a gluten-induced autoimmune disease, is associated with low bone mineral density (BMD) among children. Unfortunately, it is often diagnosed in adulthood, which may lead to an increased risk of fragile bones. The aim of this systematic review was to report on BMD status among young adults newly diagnosed with $\mathrm{CD}$, and to examine the effect of a gluten-free diet (GFD), nutritional supplements, such as vitamin D, or antiresorptive medications on BMD recovery. Databases searched were Medline, Embase, and Cochrane Library up to July 2nd, 2020. Both observational studies and clinical trials were considered, if patients were newly diagnosed and between 20 and 35 years of age and reported on BMD. We critically appraised the identified studies using ROBINS-I and summarized the findings narratively. Out of 3991 references, we identified 3 eligible studies: one cross-sectional study and two longitudinal studies. In total, 188 patients were included, and the study population consisted primarily of women with an age range between 29 and 37 years old. Compared to healthy controls, our target population had lower BMD. Moreover, a strict GFD may increase BMD during a follow-up period of up to 5 years. Newly diagnosed CD patients aged 20-35 years are at risk of lower BMD. Therefore, it may be crucial to assess BMD at time of diagnosis in young women. Whether the results can be extrapolated to young men is unknown. While strict GFD may improve BMD over time, there is a lack of robust evidence to demonstrate that nutritional supplements or antiresorptive agents are beneficial in the prevention of fragile bones in this age group.
\end{abstract}

Keywords Celiac disease $\cdot$ BMD $\cdot$ DXA $\cdot$ Osteopenia $\cdot$ Osteoporosis $\cdot$ Gluten-free diet
Abbreviations
CD Celiac disease
BMD Body mass density
BMI Body mass index

DXA Bone densitometry

GFD Gluten-free diet

RCT Randomized controlled trial
PROSPERO Registration: CRD42020191435, submitted in July 2020.

Mina Nicole Händel

mina.nicole.holmgaard.handel@ regionh.dk

Caterina Mosca

caterina.mosca91@gmail.com

Fanney Thorsteinsdottir

fanney.thorsteinsdottir@ regionh.dk

Bo Abrahamsen

b.abrahamsen@physician.dk

Jüri Johannes Rumessen

J.rumessen@youmail.dk

1 Department of Medicine, Holbæk Hospital, Holbæk, Denmark
2 Abdominalcenter K, Bispebjerg Hospital, Copenhagen, Denmark

3 Research Unit for Dietary Studies, The Parker Institute, Bispebjerg and Frederiksberg Hospital, Frederiksberg, Denmark

4 Nuffield Department of Orthopaedics, Rheumatology and Musculoskeletal Sciences, University of Oxford, Oxford, UK

5 Department of Clinical Research, Odense Patient Data Explorative Network, University of Southern Denmark, Odense, Denmark

6 Department of Gastroenterology, Herlev and Gentofte Hospital, University of Copenhagen, Herlev, Denmark 


\section{Introduction}

Celiac disease (CD) is a chronic, immune-mediated disorder characterized by malabsorption of nutrients after ingestion of the protein gluten, found in wheat, rye and barley, in genetically susceptible individuals. CD is characterized by expression of the human leukocyte antigen (HLA) class II molecules DQ2 or DQ8 involved in activating the $\mathrm{T}$ lymphocytes and initiating the autoimmune process [1]. The inflammatory process mediated by the T-cells leads to villus atrophy of the small intestinal mucosa [2], causing malabsorption [3].

$\mathrm{CD}$ can occur at any age, with heterogeneous symptoms or conditions and/or poor disease awareness, and since routine screening of $\mathrm{CD}$ is not offered to the general public, many remain undiagnosed [4].

Several extraintestinal manifestations, including anemia, neurologic symptoms, menstrual abnormalities, infertility, recurrent spontaneous abortions, growth retardation, dermatitis herpetiformis, aphthous stomatitis, dental defects, have been associated with CD [5].

Bone health can also be negatively affected in CD owing in part to the inflammatory process and in part to malabsorption of calcium and vitamin D. Osteopenia and osteoporosis and bone fractures are the most common skeletal complications associated with CD [6]. The risk of bone fractures is increased in $\mathrm{CD}$ regardless of the presence of symptoms [7]. It was shown that at baseline, CD was associated with a $30 \%$ increase [ $95 \%$ confidence interval (CI) $1.14,1.50]$ in the risk of any fracture and a $69 \%$ increase in the risk of hip fracture (95\% CI 1.10, 2.59) [8].

Progression of non-invasive methods such as bone densitometry has shown that a large proportion of patients with CD have impaired bone mass and therefore are prone to bone fractures [9].

A systematic review and meta-analysis focused on CD and bone health in children and adolescents showed that they have suboptimal bone health and shorter stature at diagnosis [10]. This could indicate that the prevalence of reduced BMD may also be high in young adult patients with CD. Suboptimal bone accumulation at the time of reaching peak bone mass (age 20-30) may influence risk of later osteoporosis [11]. Therefore, early identification of reduced BMD may improve motivation to comply with gluten-free diet and promote calcium and vitamin D supplementation to potentially reduce risk of fractures later in life.

The objective of this systematic review was to synthesize any existing literature on (1) the prevalence of osteoporosis and osteopenia in young adult patients (20-35 years old) with newly diagnosed CD, (2) the effect of a gluten-free dietary intervention, started at the time of diagnosis on BMD in newly diagnosed $\mathrm{CD}$ patients in the 20-35 age group, (3) the effect on gluten-free dietary intervention in combination with nutritional supplements, including probiotics on BMD in newly diagnosed CD patients in the 20-35 age group, and (4) the effect of anti-osteoporosis drugs on BMD in newly diagnosed CD in the $20-35$ age group.

\section{Methods}

This systematic review was conducted according to the established methods recommended by the cochrane collaboration and reported in accordance with the guidelines of preferred reporting items for systematic review and meta-analysis (PRISMA) [12]. The study protocol was pre-specified and registered in PROSPERO (ID: CRD42020191435), submitted in July 2020. The registration record was automatically published exactly as submitted. Thus, the PROSPERO team did not check eligibility, due to an exclusive focus on COVID-19 registrations during the 2020 pandemic.

\section{Eligibility Criteria}

We applied the population, intervention, comparison, and outcome (PICO) characterization [13] to operationalize the research question, and to determine the eligibility criteria. We were interested in assessing risk of osteoporosis and osteopenia in patients aged 20-35 years, and were newly diagnosed with $C D$. Studies not applying an age limit at all, were included insofar as the mean age of the participants was within our age range. The primary outcome was BMD [measured via dual-energy X-ray absorptiometry (DXA)] either reported dichotomized, as the number of patients who have lower BMD, compared to healthy controls, or as mean/median values with confidence intervals, and modification in BMD after the interventions (such as gluten-free diet, nutritional supplements and antiresorptives).

The definitions of population, intervention, comparators, outcomes, and study design are specified in Table 1.

The research question can be addressed through inclusion of case-control studies, cohort studies, and randomized controlled trials. However, to evaluate effectiveness of interventions, i.e., research question 4 , randomized controlled trials are more applicable. Therefore, chart reviews, case series, case reports, commentaries (e.g., expert opinion, consensus statements), conference abstract reporting on studies that have provided a full report elsewhere, and animal studies were excluded. 
Table 1 Population, Intervention, Comparison, and Outcome (PICO) criteria for inclusion and exclusion of studies

\begin{tabular}{|c|c|c|c|c|c|}
\hline $\begin{array}{l}\text { Research } \\
\text { question }\end{array}$ & Population & Intervention & Comparator & Outcome & Study design \\
\hline 1 & \multirow{4}{*}{$\begin{array}{l}\text { Individuals aged } \\
\text { 20-35 years newly } \\
\text { diagnosed with CD }\end{array}$} & DXA & Healthy controls & $\begin{array}{l}\text { BMD T-score at diag- } \\
\text { noses }\end{array}$ & $\begin{array}{l}\text { Case-control studies, } \\
\text { cohort studies }\end{array}$ \\
\hline 2 & & $\begin{array}{l}\text { Diet with elimination of } \\
\text { gluten }\end{array}$ & $\begin{array}{l}\text { Pre-intervention values } \\
\text { for the cohort }\end{array}$ & Change in BMD T-score & $\begin{array}{l}\text { Case-control studies, } \\
\text { cohort studies }\end{array}$ \\
\hline 3 & & $\begin{array}{l}\text { Dietary supplements in } \\
\text { addition to adherence } \\
\text { to a strict gluten-free } \\
\text { diet }\end{array}$ & $\begin{array}{l}\text { A strict gluten-free diet } \\
\text { without supplements/ } \\
\text { probiotics }\end{array}$ & Change in BMD T-score & $\begin{array}{l}\text { Case-control studies, } \\
\text { cohort studies and } \\
\text { RCTs }\end{array}$ \\
\hline 4 & & $\begin{array}{l}\text { Osteoporosis drugs (anti- } \\
\text { resorptives includ- } \\
\text { ing denosumab and } \\
\text { bisphosphonates) }\end{array}$ & Placebo & Change in BMD T-score & $\mathrm{RCTs}$ \\
\hline
\end{tabular}

$B M D$ bone mineral density, $C D$ coeliac disease, $D X A$ dual-energy X-ray absorptiometry, $R C T$ randomized controlled trials

\section{Search Strategy}

The search was conducted July 2nd 2020 by MNH. The databases we searched was Medline and Embase via Ovid, and Cochrane Library via Medline and Embase. Literature search strategies were developed using medical subject headings (MeSH) and free text. No study design, date, or language limits were imposed on the search. The full electronic search strategy for all databases are presented in detail in Supplementary Material. Reference lists of included publications and literature reviews was screened to identify additional data sources. Furthermore, we contacted experts in the field to identify additional studies (cciacci@unisa.it and bzanchetta@idim.com.ar).

\section{Study Selection}

The studies generated from the defined search strategy were imported to Endnote, where duplicates were removed. Then the remaining literature search results were uploaded to COVIDENCE Software, an Internet based software program that facilitates collaboration among reviewers during the study selection process. In Covidence, additional duplicates were identified.

References were independently screened by title and abstract against the inclusion criteria by two review authors (CM, FT). We obtained full reports for all titles that appear to meet the inclusion criteria or where there was any uncertainty. The full text-reports evaluation to determine eligibility, were independently screened by the same two review authors (CM, FT). We resolved disagreement through discussion, or if needed by involving a third review author (MNH). Publications without a full-text available were excluded. Moreover, due to language limitation we only included studies in English. We recorded the reasons for excluding trials at full-text level (reason for exclusion can be found in supplementary material). Neither of the review authors were blinded to the journal titles or to the study authors or institutions.

\section{Data Extraction}

$\mathrm{CM}$ and FT extracted data independently and resolved disagreements through discussion, with the help of MNH in case of unresolved disagreements. We extracted the generic and the trade name of the experimental intervention, the study design, the type of control used, frequency and duration of treatment, number of participants included, patient characteristics (average age, gender, duration of follow-up), funding and conflict of interest of every included full-text study.

\section{Quality Assessment}

In order to evaluate the risk of bias of non-randomized studies, two authors (CM and MNH) used ROBINS-I [14]. The tool views each study as an attempt to emulate (mimic) a hypothetical pragmatic target randomized trial and covers seven distinct domains through which bias might be introduced. The first two domains, covering confounding and selection of participants into the study, address issues before the start of the interventions that are to be compared ("baseline"). The third domain addresses classification of the interventions themselves. The other four domains address issues after the start of interventions: biases due to deviations from intended interventions, missing data, measurement of outcomes, and selection of the reported result. Our prespecified confounders were endocrine diseases (Cushing, Addison, hyperparathyroidism and Diabetes), chronic use of corticosteroids, malabsorption caused by other inflammatory bowel diseases, chronic use of alcohol and smoking. Our prespecified co-intervention was: physical activity. Discrepancies were identified and resolved through discussion 
and if consensus was not reached, a third reviewer (FT) was involved and provided input.

\section{Meta-analysis}

Although a meta-analysis was planned in the protocol, this was deemed inapplicable, due to the scarcity of the evidence.

\section{Results}

\section{Literature Search}

After deduplication in Endnote, we identified 3991 references generated from the defined search strategy, and these were imported to COVIDENCE, where further 213 duplicates were removed. Then 3778 references were screened based on their title and abstract. Here 3622 studies were found irrelevant and 156 were deemed relevant for the fulltext assessment. Of these only three studies [15-17] were eligible to be included in data extraction for the reasons summarized in the PRISMA flowchart (supplementary material).

\section{Description of Primary Studies}

We identified three relevant studies from New Zealand, Italy, and Argentina, respectively [15-17]. One was a cross-sectional study [17] and the other two were longitudinal [15, 16]. In total, 188 patients were included, and the sample size ranged from 10 to 110 . All studies enrolled women, only (Tables 2, 3).

\section{Newly Diagnosed Celiac Disease and Bone Status}

All three studies showed an association between untreated CD and reduced BMD [15-17] (Table 4). Zanchetta et al. found that premenopausal women $(n=31)$ with newly diagnosed CD at median age 29 years had significantly lower BMD z-scores at lumbar spine, femoral neck, and distal radius compared with healthy women $(n=22)$ of similar age (median age 30 years) [16]. Similarly, Lunt et al. found that $\mathrm{Z}$ score (SD units below age-appropriate mean) at the lumbar spine was -0.98 for subjects with $\mathrm{CD}(n=10)$, compared with -0.12 for subjects with only diabetes $(n=89)$ [17].

\section{Gluten-Free Diet and Long-term Bone Health}

Zanchetta et al. assessed the influence of a gluten-free diet (GFD) among newly diagnosed CD patients, showing that BMD T-score increased significantly at all regions evaluated (lumbar spine: $2.8 \%$, femoral neck: $2.5 \%$, total hip: $3.7 \%$, and distal radius: $3.7 \%$ ), compared with the same population before the intervention [16]. Passananti et al. randomly allocated 110 newly diagnosed CD patients into two groups of 55, one group where they measured BMD at diagnosis and again after 2 years on a GFD, while in the other group, the BMD was measured at diagnosis and again after 5 years on a GFD [15]. About $52 \%$ of the patients had a pathological BMD at diagnosis. At 2 years follow-up BMD at femur was higher than that at diagnosis $(p=0.04$ for the femoral neck and $p=0.037$ for Ward's triangle), an increase for the spine was observed, even though not statistically significant $(p>0.05)$. While the 5-year follow-up showed an increase of BMD in femur and spine, both higher than at diagnosis $(p=0.04)$ [15]. The improvement in bone density following a GFD was significant already after only 2 years, and even greater after 5 years, at both the femur and the spine [15] (Table 5).

\section{Results from ROBINS-I Tool Assessment}

In supplementary material the risk of bias assessment of the observational studies are presented, and the results showed that all three studies were rated as critical risk of bias in domain 1 (confounding), since none of the studies adjusted for confounders [15-17]. One study was rated with serious risk of bias in domain 2 (selection of participants) [17], because selection into the study was related (but not very strongly) to intervention and outcome. Two studies did not report clearly on domain 4 (departures from intended intervention) [15, 17], or domain 5 (missing data) [17]. The studies did not indicate bias in the selection of reported results, according to the review process. Still, since none of the studies had reported a pre-specified protocol, we rated the risk of bias to be moderate within this domain. The overall judgment of the risk of bias in all studies was rated as a critical risk of bias.
Table 2 Study identification of the included studies

\begin{tabular}{lllll}
\hline Study's first author, year & Country & Trial registration & Study design & $\begin{array}{l}\text { Conflict of } \\
\text { interest/spon- } \\
\text { sorship }\end{array}$ \\
\hline Lunt (2001) [17] & New Zealand & Not reported & Cross-sectional & Not reported \\
Passananti (2011) [15] & Italy & Not reported & Longitudinal & No conflicts \\
Zanchetta (2015) [16] & Argentina & Not reported & Longitudinal & No conflicts \\
\hline
\end{tabular}




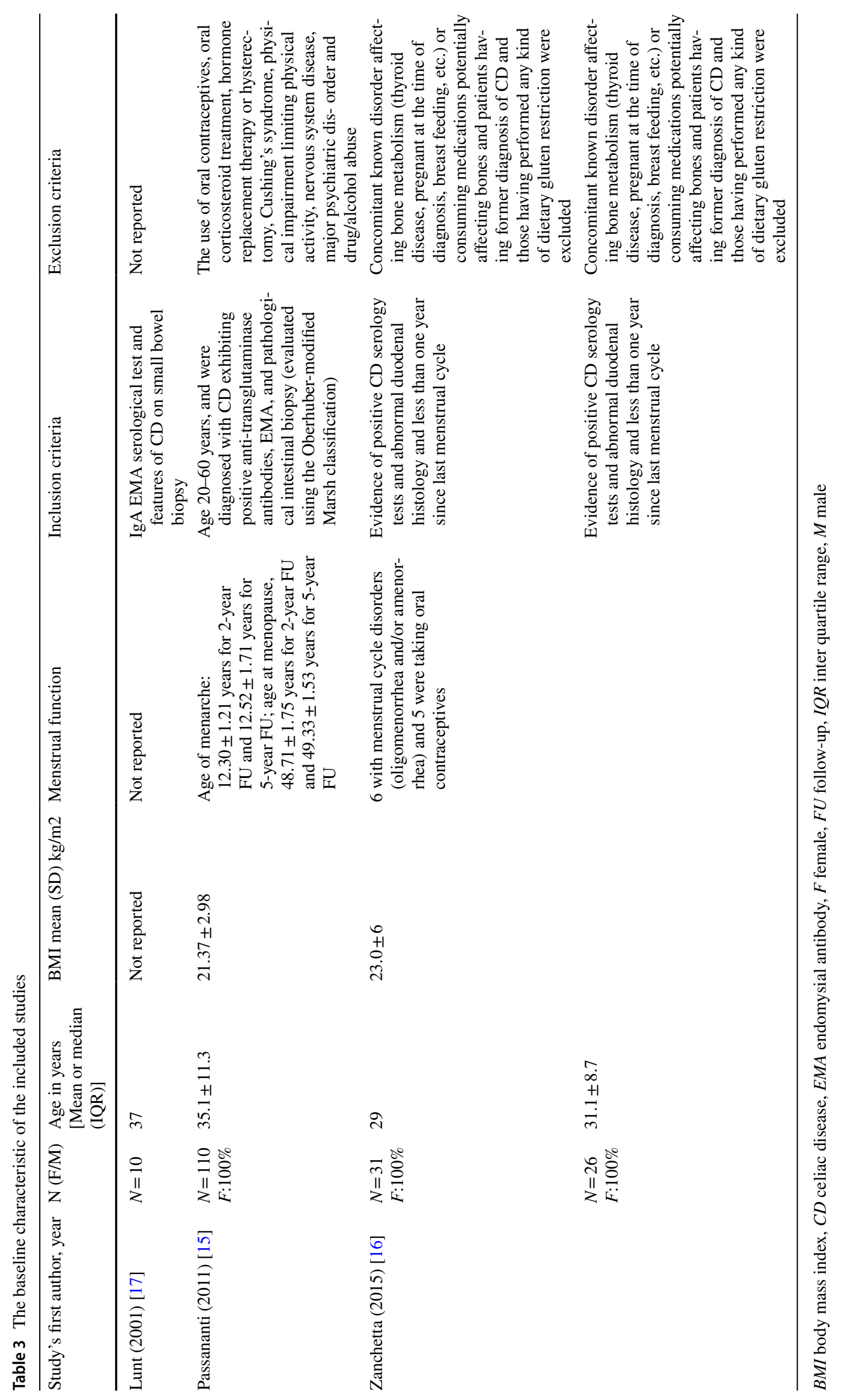


Table 4 The method, reported outcomes and the results of the included studies examining the prevalence of osteopenia and osteoporosis

\begin{tabular}{|c|c|c|c|c|c|}
\hline Study's first author, year & Method & Reported outcomes & $\begin{array}{l}\text { Prevalence of } \\
\text { osteopenia, \% }\end{array}$ & $\begin{array}{l}\text { Prevalence of osteo- } \\
\text { porosis, } \%\end{array}$ & $T$-score mean (range) \\
\hline Lunt (2001) [17] & $\begin{array}{l}\text { Bone mineral } \\
\text { densitometry } \\
\text { (DXA) }\end{array}$ & Osteopenia, \% & $4 \%$ & Data not shown & $\begin{array}{l}\text { Vertebral: }-0.98 \\
\text { Femoral: }-0.72\end{array}$ \\
\hline Zanchetta (2015) [16] & $\begin{array}{l}\text { Bone mineral } \\
\text { densitometry } \\
\text { (DXA) }\end{array}$ & $Z$-score & Data not shown & Data not shown & $\begin{array}{l}Z \text {-score: Ver- } \\
\text { tebral: }-0.50 \\
\quad(p=0.003) \\
\text { Femoral: }-0.20 \\
\quad(p=0.03)\end{array}$ \\
\hline
\end{tabular}

\section{Discussion}

\section{Summary of the Evidence of BMD Status}

Low BMD and high prevalence of osteoporosis appears typical of newly diagnosed CD among patients between 20 and 35 years, and suboptimal bone accumulation at the time of reaching peak bone mass (age 20-30) may influence risk of later osteoporosis [15, 17]. However, in our systematic review, we only identified two studies of the study population of interest [15, 17]. Even though the population consists of women only, a similar pattern was seen when also scrutinizing reports from conference abstracts [18-23], so that 30-60\% of the newly diagnosed patients aged 15-35 years were generally reported as having low BMD [18, 21, 23], and 18-35\% osteoporosis [19, $21,22]$. Similar results have also been found among newly diagnosed children and adolescents [10]. Even though the evidence is scarce and most studies of low reporting quality, these findings are sufficiently in agreement to highlight the need to routinely assess bone density in all patients with newly diagnosed CD. Unfortunately, data on young men is currently lacking. Therefore whether the findings on women can be extended to men, is at this point speculative, although it might be prudent given the previous findings in children and adolescents [10].

\section{Summary of the Evidence of Gluten-Free Diet}

From the two studies identified in the present systematic review, it seems that BMD benefits from a strict GFD. The results from the two included studies show a significant improvement already after 1 year, and even greater after 2 and 5 years $[15,16]$. These results are similar to the results presented in conference abstracts [24, 25]. For instance, Longarini et al. showed that by following a strict GFD, the one-year BMD among 31 pre-menopausal women was improved significantly at the distal radius (mean \pm SD) $(-1.94 \pm 1.27$ vs. $-1.43 \pm 1.06)$, though not at the lumbar spine level [24], and the beneficial results improve with adherence over time [20,26].

The results may be distorted due to lack of adjustments for crucial confounders, such as gonadal and menstrual function. Also, it cannot be excluded that the findings may

Table 5 The intervention, control, reported outcomes, and authors conclusion of the included studies examining the influence of gluten-free diet

\begin{tabular}{|c|c|c|c|c|}
\hline Study's first author, year & Intervention & Control & Reported outcomes & Results \\
\hline Passananti (2011) [15] & $\begin{array}{l}\text { Gluten-free diet after } 2 \text { and } \\
5 \text { years }\end{array}$ & Before intervention & BMD & $\begin{array}{l}\text { The mean BMD at femur was } \\
\text { higher than that at diagnosis } \\
\text { for the femoral neck and for } \\
\text { Ward's triangle, and no sig- } \\
\text { nificant increase in the BMD was } \\
\text { observed for the spine } \\
\text { The 5-year FU showed an increase } \\
\text { of BMD at the femur and at the } \\
\text { spine, for both being the BMD } \\
\text { higher than at diagnosis }\end{array}$ \\
\hline Zanchetta (2015) [16] & Gluten-free diet after 1 year & $\begin{array}{l}\text { Before intervention } \\
\text {-group of healthy } \\
\text { controls }\end{array}$ & BMD and T-score & $\begin{array}{l}\text { BMD, reported as } \mathrm{g} / \mathrm{cm}^{2} \text {, and DXA } \\
\text { T-score increased significantly at } \\
\text { all regions evaluated (LS: } 2.8 \% \text {, } \\
\text { FN: } 2.5 \% \text {, TH: } 3.7 \% \text {, and distal } \\
\text { radius: } 3.7 \% \text { ) }\end{array}$ \\
\hline
\end{tabular}


be partially explained by a change in body mass index (BMI) during the study period [27, 28]. Indeed, Passananti and colleagues showed that compared to time of diagnosis, an increased mean BMI was found at the 5-year follow-up $\left(21.37 \pm 2.98 \mathrm{~kg} / \mathrm{m}^{2}\right.$ vs. $\left.22.44 \pm 3.44 \mathrm{~kg} / \mathrm{m}^{2}\right)$ [15]. However, the scope of the paper is to provide a synthesis on the recovery of BMD following intervention and whether a concomitant improvement in gonadal hormones or body weight contributes to the BMD increase-though interesting from a pathophysiology angle-does not alter the overall clinical message or patient management.

\section{Summary of the Evidence of Nutritional Supplements and Antiresorptives}

We also aimed to examine the effect of a GFD combined with nutritional supplements or the effect of antiresorptive osteoporosis agents, but no studies meeting the review criteria were identified. One conference abstract was identified addressing parenteral bisphosphonate intervention [26]; while relevant to the discussion the paper is nominally outside our prespecified criteria since the population was not newly diagnosed. The authors studied 34 adult patients randomized into two groups. The first group received calcium and vitamin $\mathrm{D}$ supplementation along with GFD (standard care), while patients in the second group also received a single infusion of zoledronic acid (intervention arm). BMD at diagnosis was low in $28(82 \%)$ patients, but after 1 year, the T-score improved strongly and had a clinical significance in both groups [26]. In the standard care group $(n=15)$, the mean T-score improved from $-3.3 \pm 1.2$ to $-2.1 \pm 1.4)$ and in the intervention arm, the mean T-score improved $(n=13)$ from $-2 \pm 1.3$ to $-1.1 \pm 1.8$. However, the difference between the changes achieved in the two groups was not statistically significant. The authors conclude that zoledronic acid had a little additive effect on bone BMD compared to a GFD with calcium and vitamin D supplementation [26]. While the pilot study does not in itself offer RCT evidence that intervention is effective in restoring BMD, the absolute differences in BMD achieved were large and clinically important in both arms of the study.

\section{Strengths and Limitations of the Included Studies}

Overall, the included studies were assessed as critical risk of bias using the ROBINS-I tool [14]. According to ROBINS-I, this means that the studies are too problematic to provide any useful evidence. High-quality studies are much needed.

\section{Strengths and Limitations of Our Review}

In order to ensure high methodological quality, this systematic review followed the guidelines of the cochrane collaboration and PRISMA as well as pre-registering a protocol at PROSPERO. Moreover, a comprehensive search and duplicate full-text study selection, data extraction, and quality assessment were used. Furthermore, the authors of the included studies were contacted for further information. Amongst the limitations, we acknowledge that since we only included studies reported in English, there might be relevant studies unidentified. Moreover, unpublished papers were not searched, and thus relevant studies may have been unidentified. The review authors were not blinded in the process of selecting literature.

\section{Considerations for Further Research}

Given the high prevalence of low BMD in patients with CD [6-8], it was surprising to find an almost complete lack of studies examining the effects of dietary and/or pharmaceutical interventions that could potentially improve bone health in patients with newly diagnosed CD.

It would be helpful if subsequent RCTs would focus on patients who still had a BMD deficit after having been successfully maintained on a GFD for a reasonable period, probably one or two years, before initiation of additional intervention. Moreover, the major research gap calls for an in-depth examination of the effect of antiresorptive agents and/or nutritional supplements on BMD in young adult celiac patients. By identifying and characterizing such strategies, future recommendations among this high-risk patient group can be informed, with the short-term aim of improving peak bone mass, and with the long-term aim of lowering the prevalence of osteopenia, osteoporosis, and fractures later in life.

\section{Conclusion}

The systematic review demonstrates a high prevalence of low BMD in the 20-35 years of age population with newly diagnosed $\mathrm{CD}$, a decrease which if not corrected will be associated with an increased risk of fractures. Therefore, the results support routine assessment of BMD in young women at disease onset, but whether the findings can be extrapolated to young men is unknown. While a strict GFD was found to be beneficial to the BMD in the study population of interest, we identified a large knowledge gap regarding the potential for nutritional supplements or antiresorptive 
agents in addition to GFD to aid BMD recovery and prevent fragile bones among newly diagnosed CD patients in young adulthood.

Supplementary Information The online version contains supplementary material available at https://doi.org/10.1007/s00223-021-00938-w.

Author Contributions Conceptualization, BA and $\mathrm{MNH}$; methodology, $\mathrm{MNH}$; formal analysis, $\mathrm{CM}, \mathrm{FT}$, and $\mathrm{MNH}$; writing-original draft preparation, all authors; writing — review and editing, all authors; visualization, all authors; supervision, $\mathrm{MNH}$. All authors have read and agreed to the published version of the manuscript.

Funding This research did not receive any specific Grant from funding agencies in the public, commercial, or not-for-profit sectors. The Parker Institute, Bispebjerg and Frederiksberg Hospital, is supported by a core grant from the Oak Foundation (OCAY-18-774-OFIL).

\section{Declarations}

Conflict of interest Professor Bo Abrahamsen has received institutional research Grants from UCB, Novartis and Kyowa-Kirin, and consulting and speakers' fees from UCB, Kyowa-Kirin and Amgen, outside the current study. Dr. Mina Nicole Händel has received institutional research Grants from UCB, outside the current study. The remaining authors confirm no conflict.

\section{References}

1. van Heel DA, West J (2006) Recent advances in coeliac disease. Gut 55(7):1037-1046

2. Pellicano R, De AC, Ribaldone DG, Fagoonee S, Astegiano M (2013) 2013 update on celiac disease and eosinophilic esophagitis. Nutrients 5(9):3329-3336

3. Schuppan D, Junker Y, Barisani D (2009) Celiac disease: from pathogenesis to novel therapies. Gastroenterology 137(6):1912-1933

4. Caio G, Volta U, Sapone A, Leffler DA, De Giorgio R, Catassi C et al (2019) Celiac disease: a comprehensive current review. BMC Med 17(1):142

5. Ribaldone DG, Astegiano M, Fagoonee S, Rizzetto M, Pellicano R (2011) Epilepsy and celiac disease: review of literature. Panminerva Med 53(4):213-216

6. Larussa T, Suraci E, Nazionale I, Abenavoli L, Imeneo M, Luzza F (2012) Bone mineralization in celiac disease. Gastroenterol Res Pract 2012:198025

7. Pinto-Sanchez MI, Bai JC (2019) Toward new paradigms in the follow up of adult patients with celiac disease on a gluten-free diet. Front Nutr 6:153

8. Heikkila K, Pearce J, Maki M, Kaukinen K (2015) Celiac disease and bone fractures: a systematic review and meta-analysis. J Clin Endocrinol Metab 100(1):25-34

9. Ganji R, Moghbeli M, Sadeghi R, Bayat G, Ganji A (2019) Prevalence of osteoporosis and osteopenia in men and premenopausal women with celiac disease: a systematic review. Nutr J 18(1):9

10. Fedewa MV, Bentley JL, Higgins S, Kindler JM, Esco MR, MacDonald HV (2020) Celiac disease and bone health in children and adolescents: a systematic review and meta-analysis. J Clin Densitom 23(2):200-211

11. Boot AM, de Ridder MA, van der Sluis IM, van Slobbe I, Krenning EP, Keizer-Schrama SM (2010) Peak bone mineral density, lean body mass and fractures. Bone 46(2):336-341
12. Page MJ, McKenzie JE, Bossuyt PM, Boutron I, Hoffmann TC, Mulrow CD et al (2021) The PRISMA 2020 statement: an updated guideline for reporting systematic reviews. Int J Surg 88:105906

13. Guyatt GH, Oxman AD, Kunz R, Atkins D, Brozek J, Vist G et al (2011) GRADE guidelines: 2. Framing the question and deciding on important outcomes. J Clin Epidemiol 64(4):395-400

14. The Risk of Bias in Non-Randomized Studies-of Interventions (ROBINS-I) Assessment Tool. 2016. Available online: https:// www.bristol.ac.uk/population-healthsciences/centres/cresyda/ barr/riskofbias/robins-i/. Accessed 29 Dec 2021

15. Passananti V, Santonicola A, Bucci C, Andreozzi P, Ranaudo A, Di Giacomo DV et al (2012) Bone mass in women with celiac disease: role of exercise and gluten-free diet. Dig Liver Dis 44(5):379-383

16. Zanchetta MB, Longobardi V, Costa F, Longarini G, Mazure RM, Moreno ML et al (2017) Impaired bone microarchitecture improves after one year on gluten-free diet: a prospective longitudinal HRpQCT study in women with celiac disease. J Bone Miner Res 32(1):135-142

17. Lunt H, Florkowski CM, Cook HB, Whitehead MR (2001) Bone mineral density, type 1 diabetes, and celiac disease. Diabetes Care 24(4):791-792

18. Shelley L, Jacobs A, Daveson AJM (2016) Nutrient deficiencies are common at the time of diagnosing coeliac disease in an Australian cohort. J Gastroenterol Hepatol 31(Supplement):164

19. Baccouche K, Amri D, Bouzaoueche M, Belghali S, Zeglaoui $\mathrm{H}$, Bouajina E (2016) Low bone mineral density in patients at diagnosis of celiac disease. Osteoporos Int 1:S256-S257

20. Casella S, Zanini B, Lanzarotto F, Villanacci V, Magni A, Caselani $\mathrm{F}$ et al (2011) Clinical characteristics of celiac disease and response to gluten free diet are different in elderly and in younger patients. Dig Liver Dis 3:S234

21. Galli G, Lahner E, Conti L, Esposito G, Sacchi MC, Di Giulio $E$ et al (2017) Male gender and underweight are associated with osteoporosis in patients with new diagnosis of coeliac disease. United Eur Gastroenterol J1 5(Supplement 1):A379

22. Singh V, Jahangeer B, Singh H, Khaliq A, Bhadada SK, Vaiphei $\mathrm{K}$ et al (2011) Correlates of bone demineralization in patients with adult celiac disease. Indian J Gastroenterol 1:A29

23. Usai Satta P, Oppia F, Cabras F (2013) Prevalence of osteopenia and osteoporosis at the time of diagnosis in a series of adult celiac patients. Dig Liver Dis 2:S216

24. Longarini GI, Zanchetta MB, Costa AF, Longobardi V, Temprano MP, Vazquez $\mathrm{H}$ et al (2014) Impaired bone microstructure improves after oneyear on gluten-free diet. A prospective longitudinal study in women with active celiac disease. United Eur Gastroenterol J 1:A278

25. Papamichael K, Kokkinakis E, Archavlis EJ, Theodoropoulos I, Tzivras D, Karakoidas C et al (2010) Effect of a gluten free diet on bone mineral density in patients with celiac disease. Gastroenterology 1:S308

26. Kumar M, Bhadada S, Kochhar R, Kumar PR, Khandelwal N, Jain K et al (2010) Effect of zoledronic acid in patients with newly diagnosed adult celiac disease: a prospective, randomized study. Indian J Gastroenterol 1:A31

27. Bolland MJ, Grey A, Rowbotham DS (2016) Outcomes of bone density measurements in coeliac disease. $\mathrm{N}$ Z Med J 129(1429):40-44

28. Webster J, Vajravelu ME, Choi C, Zemel B, Verma R (2019) Prevalence of and risk factors for low bone mineral density in children with celiac disease. Clin Gastroenterol Hepatol 17(8):1509-1514

Publisher's Note Springer Nature remains neutral with regard to jurisdictional claims in published maps and institutional affiliations. 